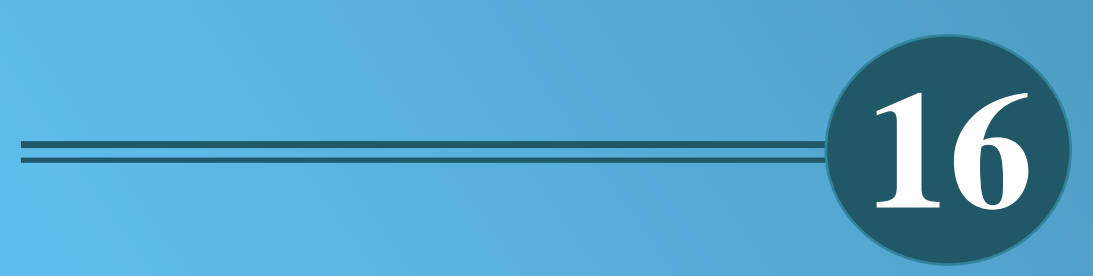

La nueva ruralidad y el turismo, una alternativa de desarrollo sostenible en Dos Mangas, provincia de Santa Elena.

Jhony Ernesto Yumisaca Tuquinga, Arnaldo Efrén Mendoza Tarabó, Jenniffer Gonzabay Rosales 


\title{
La nueva ruralidad y el turismo, una alternativa de desarrollo sostenible en Dos Mangas, provincia de Santa Elena.
}

\section{The new rurality and tourism, an alternative sustainable development in Dos Mangas, Santa Elena province.}

\author{
Jhony Ernesto Yumisaca Tuquinga ${ }^{1,}$ Arnaldo Efrén Mendoza Tarabó, Jenniffer Gonzabay Rosales \\ ${ }^{1}$ Profesor investigador de la carrera de Gestión y Desarrollo Turístico \\ Universidad Estatal Península de Santa Elena UPSE. Campus La Libertad, vía Santa Elena-La Libertad. \\ 1jyumisaca@upse.edu.ec
}

\begin{abstract}
Resumen
La actividad turística como una fuente de dinamización económica y una herramienta valiosa para salir de la pobreza, conjuntamente con la importancia que hoy en día se asigna al espacio rural, empieza a tomar un nuevo rumbo en el proceso de desarrollo de los destinos turísticos favoreciendo a la sostenibilidad de los mismos; por lo tanto, los esfuerzos de las comunidades por incursionarse en la actividad turística y minimizar la función tradicional del espacio rural, donde la producción agrícola, ganadera, de materia prima y maderera ha primado por generaciones, enfrentan nuevos retos encaminados no solo a la producción sino a la prestación de servicios, así como el de garantizar la conservación de la naturaleza y de la cultura, de tal forma que respondan al bienestar de las generaciones futuras del sector que cada vez son más endógenas. El presente artículo analiza la incursión de la comuna Dos Mangas en la provincia de Santa Elena en esta nueva oportunidad de progreso, quien propone a la nueva ruralidad con sus aspectos de multifuncionalidad, la pluriactividad y el empleo rural no agrícola como un modelo de desarrollo no tradicional. Este trabajo se desarrolló dentro del paradigma cualitativo a través del método descriptivo interpretativo y la técnica de entrevistas a actores claves tales como emprendedores, líderes locales, responsables del centro de interpretación y dirigentes de la comuna. Como resultado preliminar de la investigación, se puede afirmar que la nueva ruralidad está en proceso de convertirse en un modelo de generación de recursos a través del desarrollo turístico no convencional que puede ser aplicada en comunidades con similares características.
\end{abstract}

Palabras clave: dinamización económica, espacio rural, nueva ruralidad y sostenibilidad.

\begin{abstract}
The tourist activity as a source of economic dynamism and a valuable tool to get out of poverty, together with the importance that nowadays is assigned to the rural space, begins to take a new direction in the process of development of tourist destinations favoring Their sustainability; Therefore, the efforts of the communities to enter into tourist activity and minimize the traditional role of rural areas, where agricultural, livestock, raw material and timber production has prevailed for generations, face new challenges not only to production But also to the provision of services, as well as to ensure the conservation of nature and culture, so as to respond to the wellbeing of future generations of the sector that are increasingly endogenous. The present article analyzes the incursion of the Dos Mangas municipality in the province of Santa Elena in this new opportunity of progress, who proposes to the new rurality with its aspects of multifunctionality, alloreactivity and non - agricultural rural employment as a non - traditional. This work was developed within the qualitative paradigm through the descriptive interpretive method and the technique of interviews with key players such as entrepreneurs, local leaders, responsible for the interpretation center and commune leaders. As a preliminary result of the research, it can be said that the new rurality is in the process of becoming a model of resource generation through non-conventional tourism development that can be applied in communities with similar characteristics.
\end{abstract}

Keywords: economic revitalization, rural space, new rurality and sustainability. 


\section{1.- Introducción}

La nueva ruralidad es una corriente relativamente reciente que surge para explicar las transformaciones en el espacio rural que se han generado con la implementación de las políticas neoliberales, tanto en Europa como en América Latina (Rosas, 2009) ${ }^{1}$. Esta propuesta, en definitiva concuerda con lo que otros territorios viven y proponen frente a la globalización y a la concepción tradicional conocida como la ciudad versus el campo, que interpreta las características de cada uno de estos espacios como opuestas y en la cual la primera (ciudad) subleva a la segunda (campo) (Martinez M. , 2010) ${ }^{2}$.

Evidentemente las transformaciones que experimenta el área rural son tan obvias, que a decir de Pérez ${ }^{3}$ muchos autores coinciden en identificar este proceso de cambio como nueva ruralidad. Aunque, según Gómez ${ }^{4}$ existe una tendencia a confundir la descripción del proceso de desarrollo con el alcance de la definición de nueva ruralidad. Sin embargo, cada vez se puede notar la interdependencia entre el campo y la ciudad, es decir que la integración entre los dos sectores son más intensos.

Ante cambios constantes y demandas urgentes, la relación campo ciudad cada vez se vuelve más estrecha, en tal sentido, la revalorización de los espacios rurales es más significante; de acuerdo a Schroeder $(2012)^{5}$ las áreas rurales se vinculan a procesos de urbanización y ámbitos de residencia, turismo y recreación.

El nuevo escenario que se ha formado, permite considerar conjuntamente el territorio, los asentamientos humanos y la interrelación entre ellos. El caso de estudio que se presenta se desarrolló en la comuna Dos Mangas asentada al noreste de la provincia de Santa Elena en la costa ecuatoriana, su ubicación en las estribaciones de la cordillera Chongón Colonche hace posible la práctica de un turismo diferente y alternativo, que a decir de Félix \& Doumet $^{6}$ agrupa las modalidades de ecoturismo, aventura y rural, que en la actualidad son requeridas por los demandantes.

Lo mencionado se liga a la tendencia del nuevo turista que no requiere de un paquete turístico estandarizado con rigidez y de tipo masivo, sino que prefiere un turismo más individualizado, con un alto grado de interés de tranquilidad, de contacto con la naturaleza y la cultura, dejando atrás aquellas vacaciones pasivas por eventos más participativos.

Yumisaca J./Mendoza E./Gonzabay J.
Dentro de este escenario, se puede entender y calificar a la actividad turística de importancia trascendental por su gran aporte a las economías de la mayoría de los países del mundo, generando importantes expectativas por su crecimiento histórico, que según datos de la UNWTO (2017) ${ }^{7}$ el desplazamiento de turistas alcanzó 1.235 millones al finalizar el 2016, proyectándose alcanzar 1.800 millones en 2030, lo que implica un crecimiento de promedio anual del $3.3 \%$.

La Organización Mundial del Turismo (2015) revela que los viajes por vacaciones, esparcimiento $\mathrm{u}$ otras formas de ocio representaron el $53 \%$ en el 2014, mientras un $27 \%$ se distribuyó entre visitas a parientes y amigos, razones religiosas o peregrinaciones, tratamientos de salud, etc. afirmando que alrededor del $14 \%$ de los turistas internacionales viajan por negocios o motivos profesionales y, un $6 \%$ no especifica el motivo de visita.

Precisamente, el enfoque de este estudio se centra en determinar la incursión del destino Dos Mangas en la nueva ruralidad como un modelo diferente de desarrollo, que a decir de SENA $(2012)^{8}$ se caracteriza por la multifuncional, la pluriactividad y el empleo no agrícola.

\section{2.- Discusión Teórica}

\subsection{La nueva ruralidad (NR)}

Este enfoque denominado nueva ruralidad implica mantener y sostener la población rural en los lugares de origen, haciendo énfasis en los múltiples usos del suelo, creando diversas actividades que desarrollen los agricultores y campesinos en general, impulsando acciones económicas distintas a las desarrolladas tradicionalmente en la localidad.

La nueva ruralidad (NR) es diversa, diferente al histórico y tradicional modelo basado en la explotación agrícola; Barrera ${ }^{9}$ propone tres tipos de opciones y oportunidades en esta nueva conceptualización: la multifuncionalidad, la pluriactividad y el empleo no agrícola.

La multifuncionalidad propone mantener y sostener la población rural en los lugares de origen, haciendo énfasis en los múltiples usos del suelo, cuyos componentes deben ser mantenidos para la oferta de actividades como el turismo rural.

La pluriactividad se enfoca en las diversas y nuevas actividades que desarrollan los agricultores, considerando la tierra como fuente de actividades alternativas de producción, no 
necesariamente enmarcadas en lo agropecuario, sino desde la pluriactividad, entre ellas: la venta de productos en el propio predio con valor agregado; la recreación y turismo con posibilidades de alojamiento; granjas demostrativas, agroturismo, pesca deportiva, ecoturismo, rutas alimentarías y senderismo; los acuerdos para el mantenimiento del paisaje, producción de energía eólica y captura de $\mathrm{CO} 2$.

El empleo rural no agrícola consistente en actividades económicas desarrolladas por los hogares, distintas al empleo de su propia explotación agrícola. Estas pueden ser: la agroindustria, servicios diversos, el comercio y otros, que generen valor $y$ evite que las poblaciones migren, aportando a la diversificación de actividades.

Según Martínez \& Palafox $(2015)^{10}$, la nueva ruralidad impulsa la pluriactividad para la generación de ingresos que permite conservar el modo y relaciones de producción enmarcadas en su organización social. Bajo este precepto, el turismo se suma a las actividades tradicionales de las comunidades, las cuales se convierten en un instrumento de desarrollo rural, que basado en la participación activa de la comunidad permiten impulsar y fortalecer su identidad colectiva y regional.

Al respecto, Schroeder (2011) ${ }^{11}$ enfatiza el rol dinamizador que juegan las actividades turísticas en el desarrollo rural como una de las estrategias no tradicionales más utilizadas. Bajo esta aseveración, las posibilidades de uso de los recursos naturales y culturales en los destinos turísticos del sector rural, posibilita la dinamización de oferta, ampliando las opciones de atraer y retener al visitante más tiempo en el destino.

Ante esta situación, la modalidad de turismo rural con todas sus variantes encaja en el ámbito descrito, ya que considera elementos primordiales de la cultura, la naturaleza y las labores cotidianas de la población, que transformados en producto turístico, ofrece a los visitantes una convivencia única, disfrute del entorno y la participación en las labores tradicional de la comunidad. Todas estas actividades planificadas para el conjunto del territorio rural, permite trascender hoy en día hacia lo que se denomina la nueva ruralidad.

Este nuevo modelo orientado al aprovechamiento del territorio rural, se sustenta en funciones que deben ser asignadas a la población; la prestación de servicios, el rescate y la conservación tanto del recurso natural como cultural, el turismo y ocio, vienen a fundamentar los pilares de este modelo de desarrollo (NR).

En este contexto el desarrollo de una nueva ruralidad analiza entre otros aspectos, las nuevas características del espacio rural y su impacto a través de asignar nueva funciones en contraposición del esquema tradicional relacionado a lo rural.

Por consiguiente, el turismo es considerado como una de las actividades más viables para atenuar las condiciones de pobreza y marginación de la población rural, debido al conjunto de efectos positivos tangibles e intangibles que genera en los territorios rurales (Barrera, 2006).

La acertada orientación de las comunidades y destinos hacia este nuevo modelo y la consideración del turismo dentro de la misma, permitirá paliar entre otros aspectos, la migración hacia las ciudades, la sobrecarga a los espacios cultivables, la deforestación, el monocultivo, la proliferación de residuos, el avance de la frontera agrícola, entre otros, que intranquiliza a los habitantes rurales.

\subsection{El nuevo escenario rural}

Al analizar la situación del escenario rural se puede percatar que se hacen enormes esfuerzos para equilibrar los desfases ocasionados por la globalización y todo lo que esto implica, según IICA $(2000)^{12}$ el desequilibrio es mitigado y en algunos casos superado a través de la organización comunitaria, acceso a la educación y tecnología, que en definitiva han contribuido en algo al desarrollo local y económico global.

El verdadero contexto del área rural en el mundo implica que ésta concentra el $75 \%$ de la población aquejada de pobreza medida en dólares y, que según proyecciones del Banco Mundial ${ }^{13}$, más del $60 \%$ seguirán en esa situación en el 2025.

Los recursos del área rural son otros aspectos preocupantes de este nuevo escenario, según el Programa de las Naciones Unidas para el Medio Ambiente (PNUMA) el 8,5\% del total mundial dispone de una dotación importante de recursos, un $23 \%$ de tierras arables, un $12 \%$ de tierras de cultivo, el $17 \%$ de pastos, un $23 \%$ de bosques, el $31 \%$ de agua escorrentía y el $19 \%$ del potencial hidroeléctrico mundial. Sin embargo, acciones incorrectas del hombre son cada vez más intensas, en este sentido, la importancia de alternar la agricultura con aquellos elementos que estipula la nueva ruralidad, implica una oportunidad de desarrollo para las poblaciones. 
La necesidad de superar los impactos negativos del escenario rural actual, conlleva a que los actores locales, regionales, nacionales e incluso organismos internacionales presten sus contingentes y den respuestas a exigencias propias y foráneas. En este sentido, una oportunidad es el creciente interés por la conservación de los recursos naturales y culturales de las zonas rurales, lo que estratégicamente debe ser aprovechado con propuestas, productos y servicios no tradicionales acoplados a las ventajas comparativas que presentan los espacios rurales.

Bajo esta perspectiva, se generan oportunidades para actores locales que se sumergen en estos escenarios conocidos como la nueva ruralidad porque permite mejoras en la calidad de vida. A decir de IICA (2000), el desarrollo rural sostenible en el marco de una nueva lectura de la ruralidad, ofrece a los productores agrícolas grandes posibilidades de participación, fuertes compromisos y responsabilidades.

Paradójicamente, los habitantes de las ciudades eligen los espacios rurales como lugares de residencia, ocio y turismo; mientras los campesinos comprenden que la actividad turística es una oportunidad para salir de la pobreza, por lo que el rescate y la conservación de los recursos, así como la generación de nuevos servicios se vuelven primordiales.

En tal sentido, es de interés investigar el caso de Dos Mangas, con el objeto de analizar su comportamiento bajo este enfoque.

\section{3.- Materiales y métodos}

La investigación se desarrolló dentro del paradigma cualitativo a través del método descriptivo - interpretativo, trabajo in situ identificando actores, actividades y nuevos enfoques de uso del territorio dentro de esta comuna, basado en la aplicabilidad de la técnica de entrevistas.

\section{Resultados}

\subsection{La nueva ruralidad en Dos Mangas}

Aunque los conceptos sobre la nueva ruralidad aún siguen en discusión, esta toma diferentes perspectivas según el campo disciplinario desde el cual se discute (Schroeder \& Formiga, 2009) ${ }^{14}$.

Es importante entonces tratar la nueva ruralidad desde la perspectiva turística, partiendo de la ocupación que del espacio rural hace la población agrícola y aquella vinculada a la actividad turística. Con el alcance de este concepto de nueva

Yumisaca J./Mendoza E./Gonzabay J. ruralidad, se explican los nuevos enfoques de uso del territorio de esta comuna.

Desde sus inicios Dos Mangas incursionó en actividades agrícolas, siendo esta la principal fuente de desarrollo económico y la única en cuanto al uso de suelos se refiere, esta presión sobre el espacio físico fue la causante de desequilibrios en el plano ambiental y social, la primera debido a la deforestación y avances en la frontera agrícola, mientras que la segunda se relaciona con el éxodo vivido.

Sin embargo, el crecimiento evidente de la población, la globalización, las políticas estatales y la necesidad de mejorar las condiciones de vida, motiva la incursión en un nuevo modelo de desarrollo, basado en sus recursos naturales y culturales $\mathrm{y}$, en complicidad con ONGs y organismos estatales se ha evidenciado cambios con enfoques conservacionistas tanto ambiental y social.

Producto de estos esfuerzos, el uso del territorio rural de Dos Mangas toma otro enfoque distinto al tradicional, es la década de los noventa donde se inicia la recuperación de los suelos mediante reforestación con especies propias, la dedicación de los moradores a otras actividades no agrícolas como las artesanías, la dotación de espacios para actividades de recreación y turismo, así como la prestación de servicios de hospedaje y alimentación.

Precisamente, este cambio en el área rural genera nuevas oportunidades en el área urbana, a la medida de las necesidades de los unos y las posibilidades de los otros, la oferta de hospedaje, alimentación y distracción ha crecido paulatinamente muy lejos de la típica producción acostumbrada en esta área.

Bajo estas perspectivas de nueva ruralidad, el éxodo de la población local se recompensa con el retorno de habitantes de sitios urbanos, que mitiga el despoblamiento que en este sector incurrió, por otro lado, la recuperación de 2800 hectáreas de bosque crea un marco perfecto para la actividad turística en la modalidad de turismo rural.

En definitiva, la función tradicional del espacio rural de Dos Mangas basada en la producción agrícola, ganadera y extracción de materia prima, en la que históricamente se ha centrado su economía, de apoco están cambiado. En la actualidad, la población se está direccionando hacia nuevas funciones, opacando la producción tradicional y generando la prestación de servicios que a su vez garantiza la conservación de los recursos y el bienestar de su población. 


\subsection{La nueva ruralidad y el turismo en Dos Mangas.}

A pesar de la incursión en este modelo de nueva ruralidad, aún son notables las falencias resultantes de las inexperiencias, desconocimiento, y esfuerzos individuales, sin embargo, el enfoque en el capital rural y en los esfuerzos de desarrollo endógeno de sus pobladores sería la solución a estos inconvenientes. En tal sentido, se ha considerado el contingente de capital físico, natural, cultural, humano y social, que apoyado en la nueva ruralidad permita la diversificación a través de emprender iniciativas novedosas como el turismo, las artesanías y otros complementarios, que han servido para aprovechar las oportunidades extraordinarias del entorno local, nacional e internacional.

En Dos Mangas, lo rural ya no es sinónimo de explotación agrícola y ganadera, todo lo contrario, esta comuna cuenta con actividades diversas que sobresalen de lo común; varios servicios fortalecen la actividad turística y permite la reactivación económica del medio rural de este poblado, ligándose a la conceptualización dada por Pérez (2001), en la que manifiesta que el medio rural es "el conjunto de actividades diversas pequeñas y medianas, de comercio y servicios".

Con este enfoque, el turismo en Dos Mangas se desarrolla como parte de la nueva ruralidad, fortaleciéndose en la organización comunitaria, la curva de aprendizaje sobre la actividad turística, la tecnología y el enfoque de sostenibilidad. El turismo en el espacio rural de esta comuna se debe al apoyo de Fundación Natura, Ministerio de Turismo del Ecuador, USAID, GAD provincial, entre otros, que mediante sinergia en el planteamiento y la gestión, ha conllevado a identificarse en la etapa de implicación dentro del ciclo de vida del destino.

Es decir, los determinantes para el desarrollo del turismo en el espacio rural de Dos Mangas es la cooperación, el recurso humano y la visión que se ha formado sobre esta actividad, tal como asegura Barthe \& Baldellon ${ }^{15}$ para explicar que la transformación de los espacios rurales se deben a aspectos como la capacidad de cooperación de las instituciones o la cualificación de los recursos humanos.

En todo caso, el accionar de los involucrados en el proceso turístico apunta a la calidad del mismo y mejora el acercamiento entre el medio rural y el urbano, por lo que es necesario establecer estrategias bien definidas enfocadas en

Yumisaca J./Mendoza E./Gonzabay J. necesidades puntuales de los actores, tal como afirma Acosta (2008) ${ }^{16}$ que es necesario generar sinergias y dinamizar el territorio, dando como resultado regiones competitivas e innovadoras por los productos o servicios ofrecidos.

Bajo este análisis, se denota que el dinamismo del área rural de Dos Mangas se fortalece con la incursión de nuevas actividades económicas no tradicionales, por lo que, la actividad turística dentro de este territorio debe ser el eje que dinamice el desarrollo en general de la comuna, considerando entonces al turismo rural como la modalidad más apropiada que se sustenta en los recursos existentes y en el capital más valioso, el humano.

La nueva ruralidad en Dos Mangas se manifiesta a través de la generación de oportunidades que demuestra el cambio de lo tradicional al nuevo modelo de desarrollo rural. En definitiva se evidencia en los nuevos usos del suelo dedicados a la conservación, recreación, senderismo, fincas demostrativas, cabalgatas, espacios para camping, deportes de aventura, recreación acuática, entre otros, demostrando con esto que su población ha optado por la multifuncionalidad de su espacio territorial.

Se puede evidenciar también las nuevas actividades que hacen los agricultores dentro de sus fincas, logrando adentrarse en la pluriactividad como una alternativa de desarrollo y sustento económico, donde surgen las ventas de productos en el propio predio, la aparición de espacios dedicados para la recreación, donde se ofrecen alojamiento y alimentación; otros han optado por hacer demostraciones de sus actividades agrícolas.

Bajo esta misma perspectiva de fortalecer la pluriactividad, se han formado 24 guías capacitados que ejercen la actividad de intérpretes como tarea adicional a sus acciones cotidianas, por otro lado, existen 10 personas más dedicadas al alquiler de caballos para los turistas que deseen hacer cabalgatas.

En este escenario, el empleo rural no agrícola también juega un papel preponderante en las economías de los hogares de esta comuna, las actividades incursionadas por los moradores son las relacionadas con las artesanías en paja toquilla que ejercen las mujeres y otros desarrollados en tagua a cargo de los hombres, en total se ha generado oportunidad para aproximadamente 30 personas que se dedican a estas actividades, cuyos productos son comercializados en la localidad y fuera de ella. 
El sector hostelero ha generado empleo para 37 personas durante todo el año, aunque aparentemente es mínimo, resulta ser altamente significativo para las condiciones económicas de la comuna.

Es evidente que Dos Mangas encaja en el modelo de nueva ruralidad, que ha tomado como eje fundamental el turismo rural que oferta vivir experiencias y motiva a los demandantes a dejar momentáneamente la ciudad para movilizarse y vivirlas en estos espacios. Desde este enfoque, el área rural de esta comuna es valorizada para estos efectos, convirtiéndose en un destino obligado para el ocio, el turismo, aprendizaje cultural y otras posibilidades que este destino ofrezca, pues, su valor radica en su fortaleza paisajística y recursos culturales, que según Hohl \& Tisdell (1995); Urry (2002), citados por Cawley \& Gillmor $(2008)^{17}$ son comportamientos que responden a la búsqueda posmoderna de un antídoto para la armonía de la vida urbana.

El turismo bajo la tipología rural es el eje que promueve la transformación de la comunidad en un destino rural en etapa de implicación dentro de su ciclo de vida. Para comprender mejor lo aseverado, es necesario indicar que el turismo rural son todas las actividades relacionadas con la prestación de servicios a visitantes tanto nacionales como foráneos. Tal como menciona Cabrini $(2002)^{18}$ al turismo rural como el conjunto de actividades que se desarrollan en el espacio rural y que van más allá del mero alojamiento.

Sobre la base de este concepto, el turismo rural que se practica en esta comuna es una herramienta para revitalizar la cultura local y con ella aspectos de gastronomía, tradiciones, leyendas y costumbres, sin desmerecer la puesta en valor del espacio rural a través de rutas especificas dentro de su patrimonio rural.

Tal como señala la SECTUR $(2000)^{19}$, el turismo rural se basa en el uso planificado de los recursos con el objetivo de aumentar el bienestar general de la comunidad local y del propio visitante. En todo caso, hay que dejar claro que en Dos Mangas esta modalidad es complementaria al estilo de vida generacional y está ligada a los objetivos económicos y de conservación.

\section{Discusión de los resultados}

Al realizar el análisis sobre la teoría de la nueva ruralidad, se alega que es excelente como un nuevo modelo para el desarrollo de las comunidades rurales, ya que orienta elementos muy viables para distintas realidades geográficas y culturales. Sin embargo, si los destinos turísticos

Yumisaca J./Mendoza E./Gonzabay J. no poseen el compromiso de orientar las actividades hacia la sostenibilidad y lograr sinergia entre sus actores, difícilmente se alcanzará el desarrollo y la competitividad en la localidad.

En el caso concreto de Dos Mangas, se evidencia un interés por hacer del turismo una actividad dinamizadora de la economía local, para cuyo efecto se ha organizado un comité, se ha designado una cantidad de recursos económicos, se ha capacitado a los implicados directos, se ha hecho conciencia de la conservación de los recursos existentes y se está adoptando una cultura turística.

El paso del modelo tradicional a la nueva ruralidad, se evidencia en el uso diverso del suelo comunal, en las actividades complementarias al agrícola que realizan los comuneros y en los nuevos emprendimientos relacionados con el servicio; lo que indica, que la nueva ruralidad está dando sus primeros pasos en Dos Mangas.

\section{Conclusiones}

La creciente actividad turística con enfoque sostenible y endógeno es un modelo replicable en destinos aledaños con las mismas características de ruralidad.

La nueva tipología de turismo rural que se desprende del típico turismo de masas desarrolladas en la Península, compromete a las autoridades a prestar atención y formular políticas para el fortalecimiento de la misma.

La nueva ruralidad contribuye en la generación de recursos a través del desarrollo turístico no convencional para el resto de comunidades alejadas de la zona costera, como el caso de Dos Mangas.

Si bien es cierto en Dos Mangas existe indicios de respeto ambiental, el destino aun no muestra fortaleza en responsabilidad social y una cultura turística.

Dos Mangas ha sido observada e impulsada por diversos organismos como una comunidad modelo que ha incursionado en una nueva modalidad de turismo en Santa Elena. Sin embargo, es necesario fortalecer esta inmersión del modelo basado en lo agrícola a la nueva ruralidad basado en actividades distintas, tomando como eje central el turismo rural.

El destino evidencia claramente que ha sabido aprovechar el capital rural en actividades turísticas, lo que ha permitido avanzar hasta la 
etapa de implicación en el ciclo de vida, demostrando que la pluriactividad, la multifuncionalidad y el empleo no agrícola es dable si se aprovechan las capacidades, recursos y esfuerzos propios, es decir, si se quiere desarrollar endógenamente.

La Nueva Ruralidad provee a Dos Mangas una visión distinta de desarrollo, donde están surgimiento nuevas modalidades económicas, ecológicas, de gestión, de organización y de autonomía, que presentan un desarrollo combinada entre actividades tradicionales con innovaciones que posibilitan una mejora en sus niveles de vida.

\section{Bibliografía}

Acosta, I. (2008). Revista Electrónica Zacateca sobre Pobñación y Sociedad $N^{\circ} 38$. The paradigm of new rurality as the axis of public policies. What can we expect? Obtenido de http://mpra.ub.unimuenchen.de/10042/

Barrera. (2006). Nueva ruralidad y empleo no agricola. Obtenido de http://www.cinterfor.org.uy/public/span ish/region/ampro/cinterfor/publ/barrera/ index.htm

Cabrini, L. (21 de Octubre de 2002). Turismo, desarrollo y sostenibilidad. Organiación Mundial de Turismo, VII Congreso AECIT, Jaén España . Obtenido de http://www.unwto.org/regional/europe/ PDF/SPEECHES/2002/jaen\%20_spain_ pdf

Cawley, M., \& Gillmor, D. (2008). Integrated rural tourism. Concepts and practice. Annals of tourism research. $\mathrm{Vol} 35 \mathrm{~N}^{\circ} 2$

Gómez, S. (17 de Octubre de 2001). ¿Nueva Ruralidad? un aporte al debate. Obtenido de Estudos Sociedade e Agricultura,: http://bibliotecavirtual.clacso.org.ar/ar/li bros/brasil/cpda/estudos/dezessete/ omez17.htm

IICA, I. I. (2000). El desarrollo rural sostenible en el marco de una nueva lectura de la ruralidad. Nueva Ruralidad. Panamá: IICA.

Martinez, A. P. (2015). Turismo y nueva Ruralidad: camino a ls sustentabilidad social. Letras Verdes: Revista
Latinoamericana de estudios Socioambientales.

Martinez, M. (2010). Nueva Ruralidad, la "remake" del termino pluriactividad. Revista crítica de Ciencias Sociales y Jurídicas.

Mundial, B. (2000). Estimaciones de umbral de pobreza.

Pérez, E. (2001 ). Hacia una nueva visión de lo rural: una nueva ruralidad en ämerica latina. Buenos Aires: Clacso.

Rosas, M. (Enero de 2009). Eumed. net. Biblioteca virtual de derecho, economia y ciencias sociales. . Recuperado el 17 de febrero de 2016, de http://www.eumed.net/librosgratis/2010a/647/Ruralidad $\% 20 y \% 20 \mathrm{~N}$ ueva\%20Ruralidad.htm

Schroeder, \& Formiga. (6 de Noviembre de 2009). Las actividades turístico-recreativas como dinamizadoras de centros urbanos pequeños y áreas rurales. El caso del área de influencia de Bahía Blanca. X Jornadas Argentinas de Estudios de Población Catamarca. Argentina . Obtenido de http://www.produccion.fsoc.uba.ar/aepa /xjornadas/pdf/adhoc01.pdf

Schroeder, R. (2012). El turimo rural como estrategia de dinamización territorial. el caso del sudoeste bonaerense. Anales de Geografía , 371 .

SCHROEDER, R. F. (2011). Oportunnidades para el desarrollo local: caso del Sudoeste Bonaerense (Argentina). . Cuadernos de Geografía, Revista Colombiana.

SECTUR. (2000). Programa Argentino de Turismo Rural. RAICES, 51.

SENA. (2012). El espacio rural y las nuevas posibilidades. En SENA. Bogota : SENA.

UNWTO, O. M. (2015). Panorama del turismo internacional . 